\title{
不同株型小麦干物质积累与分配对氮肥响应的动态分析
}

\author{
李国强汤亮张文宇曹卫星朱 艳”
}

南京农业大学 / 江苏省信息农业高技术研究重点实验室, 江苏南京 210095

摘 要: 为了揭示株型和施氮量对小麦干物质积累与分配动态的影响, 通过实施不同株型小麦品种和氮肥处理的田 间试验, 于主要生育期测定了各处理单株及不同器官干物质积累量, 并分别利用 Richards 和 VP 方程对其进行拟合。 结果表明, 适量施氮提高了各株型小麦的干物质平均增长速率 $\left(R_{\mathrm{a}}\right)$ 和最大增长速率 $\left(R_{\max }\right)$, 缩短了各株型小麦到达 $R_{\max }$ 的时间，延长了各株型小麦的缓增持续期 $\left(D_{3}\right)$ 。施氮提高了紧凑型矮秆品种矮抗 58 、松散型品种淮麦 17 和中间 型品种扬麦 12 的起始生长势 $\left(R_{0}\right)$, 缩短了上述 3 种株型小麦的渐增持续期 $\left(D_{1}\right)$, 降低了其到达 $R_{\max }$ 时的干物质积累量 $\left(W_{R \max }\right)$, 而紧凑型高秆品种宁麦 9 号的 $R_{0} 、 W_{\mathrm{Rmax}}$ 和 $D_{1}$ 与上述 3 种株型小麦的变化趋势相反。随施氮量的增加, 矮 抗 58 和宁麦 9 号的快增持续期 $\left(D_{2}\right)$ 呈下降趋势, 而淮麦 17 和扬麦 12 的 $D_{2}$ 以中氮处理 $\left(150 \mathrm{~kg} \mathrm{hm}^{-2}\right)$ 最低。施氮降低 了淮麦 17 和扬麦 12 的叶、穗最大分配比例 $\left(P_{\max }\right)$ 以及矮抗 58 和宁麦 9 号的茎鞘最大分配比例 $\left(P_{\text {Smax }}\right)$, 但增加了矮抗 58 和宁麦 9 号的叶部和穗部 $P_{\max }$ 以及淮麦 17 和扬麦 12 的 $P_{\text {Smax。 }}$ 施氮降低了宁麦 9 号、淮麦 17 和扬麦 12 的叶分 配比例最大下降速率及矮抗 58 和宁麦 9 号的穗分配比例最大增长速率, 而增加了矮抗 58 的叶分配比例最大下降速 率及淮麦 17 和扬麦 12 的穗分配比例最大增长速率, 但过量施氮抑制了宁麦 9 号穗分配比例最大增长速率的增加和 扬麦 12 穗分配比例最大增长速率的下降。施氮对各株型小麦茎鞘分配比例最大增长和下降速率 $\left(R_{\mathrm{Simax}}\right.$ 和 $\left.R_{\mathrm{Sdmax}}\right)$ 的影 响无明显规律。因此，在建立高产小麦栽培技术体系时，应充分考虑到不同株型小麦干物质积累和分配动态对施氮量 的响应差异。

关键词: 小麦; 株型; 施氮量; 干物质; 积累与分配; 动态分析

\section{Dynamic Analysis on Response of Dry Matter Accumulation and Parti- tioning to Nitrogen Fertilizer in Wheat Cultivars with Different Plant Types}

\author{
LI Guo-Qiang, TANG Liang, ZHANG Wen-Yu, CAO Wei-Xing, and ZHU Yan ${ }^{*}$ \\ Jiangsu Key Laboratory for Information Agriculture / Nanjing Agricultural University, Nanjing 210095, China
}

\begin{abstract}
Biomass accumulation and partition in different plant organs affect the grain yield in cereal crops. Many investigators have established equations to simulate the dynamic growth and biomass accumulation of crops in various experiments using polynomial, Expolianear, Logistic, and Richard models. However, these models have limitations in some extent. The vapor pressure (VP) model has been used to quantitatively simulate the dynamic accumulation of dry matter in maize (Zea mays L.) roots and the branch number of princess flower. In this study, the VP model was introduced in wheat (Triticum aestivum L.) to test its applicability in simulating accumulation and partitioning of biomass. Four wheat cultivars with four plant types, respectively, were planted in 2006-2007 and 2007-2008 growing seasons under the nitrogen application levels of 75, 150, and $225 \mathrm{~kg} \mathrm{ha}^{-1}$. Based on the dry weights of various organs at main growth stages, the Richards and VP equations tested the fitness of the biomass accumulation and partitioning in relation to accumulated growing-degree days. The results showed that with increasing nitrogen rate, the average dry matter growth rate $\left(R_{\mathrm{a}}\right)$, maximum growth rate $\left(R_{\max }\right)$ and duration of the third phase $\left(D_{3}\right)$ increased, while the time reaching to $R_{\max }$ was shortened consistently in four cultivars. In Aikang 58 (compact-short type), Huaimai 17 (loose type) and Yangmai 12 (intermediate type), the initial growth potential $\left(R_{0}\right)$ increased with the promotion of nitrogen rate, while the duration of early increment phase $\left(D_{1}\right)$ and the dry matter accumulation at $R_{\max }\left(W_{\mathrm{Rmax}}\right)$ decreased. In contrast, the $R_{0}, W_{\mathrm{Rmax}}$, and $D_{1}$ of
\end{abstract}

本研究由教育部新世纪优秀人才支持计划项目(NCET-08-0797), 国家自然科学基金项目(30871448), 国家高技术研究发展计划(863计划)项目 (2006AA10Z219, 2006AA10A303)和江苏省自然科学基金项目(BK2008330)资助。

*通讯作者(Corresponding author): 朱艳, E-mail: yanzhu@njau.edu.cn; Tel: 025-84396565

Received(收稿日期): 2009-04-17; Accepted(接受日期): 2009-07-25. 
Ningmai 9 (compact-high type) exhibited an opposite pattern. In Aikang 58 and Ningmai 9, the second phase duration $\left(D_{2}\right)$ decreased when the nitrogen rate increased, and Yangmai 12 and Huaimai 17 had the smallest $D_{2}$ value under the medium nitrogen rate. With the increase of nitrogen rate, the maximum dry matter partitioning percentage $\left(P_{\max }\right)$ of leaf and spike in Huaimai 17 and Yangmai 12 as well as the maximum dry matter partitioning percentage of stem and sheath $\left(P_{\text {Smax }}\right)$ in Aikang 58 and Ningmai 9 decreased. In contrast, the $P_{\max }$ of leaf and spike in Aikang 58 and Ningmai 9, and the $P_{\text {Smax }}$ in Huaimai 17 and Yangmai 12 increased accompanying with the increase of nitrogen rate. In terms of the maximum decreasing rate of dry matter partitioning percentage (MDRP) to leaf, Ningmai 9, Huaimai 17 and Yangmai 12 showed a decrease trend when nitrogen rate increased, while Aikang 58 was in an increase tendency. For the maximum increasing rate of dry matter partitioning percentage (MIRP) to spike, Aikang 58 and Ningmai 9 had a negative response to nitrogen rate and Huaimai 17 and Yangmai 12 had a positive response. Excessive application of nitrogen had negative effects on the promotion of MDRP to spike in Ningmai 9 and the reduction of MIRP to spike in Yangmai 12. No consistent effects were observed on the maximum changing rate of dry matter partitioning percentage to stem and sheath $\left(R_{\mathrm{Simax}}\right.$ and $\left.R_{\mathrm{Sdmax}}\right)$. Thereby, plant types of wheat cultivars should be considered in the nitrogen application regime in practical system of cultivation techniques.

Keywords: Wheat; Plant type; Nitrogen Rate; Dry matter; Accumulation and partitioning; Dynamic analysis

干物质是作物光合作用产物的最终形态, 其积 累、分配及运转与经济产量的形成密切相关 ${ }^{[1]}$, 而群 体光截获量和光能转运效率的调节是干物质生产的 关键 ${ }^{[2-4]}$ 。株型和氮素是影响作物群体光分布和光合 特性的重要因素 ${ }^{[4-5]}$, 而氮素又是调控小麦株型的最 有效手段 ${ }^{[6]}$ 。因此, 了解氮素对不同株型小麦的干物 质积累与分配动态的影响, 有利于明确栽培措施和 株型对小麦产量形成的调节作用和机制。株型主要 是通过调节冠层形态结构来影响冠层光分布和光截 获 ${ }^{[7-9]}$, 从而对干物质生产造成影响, 而氮素主要是 通过影响群体叶面积指数和光能转运效率来调节干 物质生产 ${ }^{[4]}$ 。与此同时, 氮素施用又对冠层形态结构 具有显著影响, 尤其是在高产水平下。Hunt 等 ${ }^{[10]}$ 及 任正隆和李尧权 ${ }^{[12]}$ 用三次多项式对干物质积累动态 进行了拟合, 但由于多项式完全是经验性的, 其方 程参数没有明确的生物学意义, 不适合植株生长的 定量分析 ${ }^{[13]}$; Goudriaan 等 ${ }^{[14]}$ 提出的 Expolianear 方程, 只适合植株发育早期到开花期间的干物质积累动 态 ${ }^{[15]}$; 随后, Royo 等 ${ }^{[16]}$ 提出具有 5 个参数的不对称 Logistic 单峰曲线, 拟合小黑麦二叶期到生理成熟期 间的干物质积累动态, 效果较好; 周竹青 ${ }^{[17]}$ 、张利 等 ${ }^{[11]}$ 以及秦舒浩等 ${ }^{[18]}$ 使用 Logistic 方程描述了干物 质积累动态, 但有学者发现, Logistics 方程可塑性较
差 ${ }^{[19]}$, 且模型参数的生物学解释存在困难 ${ }^{[20]}$ 。随后, Yang 等 ${ }^{[21]}$ 和杨义群等 ${ }^{[22]}$ 认为 Richards 更适合描述 S 形生长曲线。王空军等 ${ }^{[23]}$ 和 Faravani 等 ${ }^{[24]}$ 应用蒸汽 压力模型(vapor pressure model, VP)分别拟合了玉米 根系干物质积累动态和蒂牡丹 (princess flower)分支 节数的变化, 但 VP 方程能否较好地拟合干物质分 配比例动态, 目前尚未见文献报道。尽管已有很多 研究报道氮素和株型对小麦干物质生产的影响, 但 氮素对不同株型小麦干物质积累与分配动态影响的 报道较少, 而利用有生物学意义的数学模型对其进 行定量分析的报道更少。本研究以不同株型小麦品 种为对象, 选用 Richards 方程和 VP 方程分别拟合不 同施氮条件下小麦干物质积累特征及其在不同器官 中的分配动态, 并利用推导出的特征参数对其动态 过程进行定量分析, 以期为小麦超高产栽培提供理 论依据和技术途径。

\section{1 材料与方法}

\section{1 试验材料}

从收集的小麦品种中，根据茎藍夹角和田间长 相, 篮选出 4 个代表性小麦品种, 即紧凑型高秆宁 麦 9 号、紧凑型矮秆矮抗 58、中间型扬麦 12 和松散 型淮麦 17 (表 1)。

表 1 小麦品种株型特性

Table 1 Plant type characteristics of wheat cultivars

\begin{tabular}{llccccc}
\hline $\begin{array}{c}\text { 品种 } \\
\text { Cultivar }\end{array}$ & \multicolumn{1}{c}{$\begin{array}{c}\text { 株型 } \\
\text { Plant type }\end{array}$} & $\begin{array}{c}\text { 株高 } \\
\text { PH }(\mathrm{cm})\end{array}$ & $\begin{array}{c}\text { 旗叶长 } \\
\text { FLL }(\mathrm{cm})\end{array}$ & $\begin{array}{c}\text { 旗叶宽 } \\
\text { FLW }(\mathrm{cm})\end{array}$ & $\begin{array}{c}\text { 旗叶基角 } \\
\text { ABSL }\left(^{\circ}\right)\end{array}$ & $\begin{array}{c}\text { 茎藍角 } \\
\text { ABST }\left(^{\circ}\right)\end{array}$ \\
\hline 宁麦 9 号 Ningmai 9 & 紧凑型高秆 Compact-high & $80-85$ & 23.0 & 1.5 & 26.9 & 17.5 \\
矮抗 58 Aikang 58 & 紧凑型矮秆 Compact-short & $70-75$ & 19.9 & 1.8 & 18.8 & 16.3 \\
淮麦 17 Huaimai 17 & 松散型 Loose & $85-90$ & 25.8 & 1.5 & 29.0 & 33.3 \\
扬麦 12 Yangmai 12 & 中间型 Intermediate & $90-95$ & 18.6 & 1.7 & 20.3 & 26.3 \\
\hline
\end{tabular}

PH: plant height; FLL: flag leaf length; FLW: flag leaf width; ABSL: angle between stem and the base of flag leaf; ABST: angle between stem and tiller. 


\section{2 试验设计}

江苏省南京市农林局江宁试验站, 土壤质地为 黄棕壤, 含有机质 $14.30 \mathrm{~g} \mathrm{~kg}^{-1}$ 、全氮 $1.10 \mathrm{~g} \mathrm{~kg}^{-1}$ 、速 效磷 $10.36 \mathrm{mg} \mathrm{kg}^{-1}$ 、速效钾 $82.5 \mathrm{mg} \mathrm{kg}^{-1} ; 2006$ 年 11 月 10 日播种, 基本苗为 $1.8 \times 10^{6} \mathrm{hm}^{-2}$, 行距 $25 \mathrm{~cm}$, 前茬为水稻。南京农业大学江浦试验站, 土壤质地 为黄棕壤, 含有机质 $6.43 \mathrm{~g} \mathrm{~kg}^{-1}$ 、全氮 $0.88 \mathrm{~g} \mathrm{~kg}^{-1}$ 、 速效磷 $13.94 \mathrm{mg} \mathrm{kg}^{-1}$ 、速效钾 $151 \mathrm{mg} \mathrm{kg}^{-1}$ 。2007 年 11 月 5 日播种, 基本苗 $1.65 \times 10^{6} \mathrm{hm}^{-2}$, 行距 $25 \mathrm{~cm}$, 前茬为玉米。试验均采用裂区设计, 以 3 个施氮水 平为主区, 施纯氮 $75 、 150 、 225 \mathrm{~kg} \mathrm{hm}^{-2}$, 分别以 N75、N150、N225 表示; 4 个小麦品种为副区。3 次 重复, 随机区组排列。小区宽 $4 \mathrm{~m}$, 长 $4.5 \mathrm{~m}$ 。每个 小区均在播种前施磷肥 $\left(\mathrm{P}_{2} \mathrm{O}_{5}\right) 120 \mathrm{~kg} \mathrm{hm}^{-2}$, 钾肥 $\left(\mathrm{K}_{2} \mathrm{O}\right) 135 \mathrm{~kg} \mathrm{hm}^{-2}$ 。氮肥基追比为 $1: 1$, 追肥于拔节 期施用, 磷钾肥全部作基肥施入。其他管理措施同 高产大田栽培。

\section{3 测定项目及方法}

在小麦关键生育时期, 于每小区取样，根据植 株的器官发育情况, 将样品植株分离为叶片、茎鞘、 颖壳和籽粒, 在 $105^{\circ} \mathrm{C}$ 杀青后, 于 $80^{\circ} \mathrm{C}$ 烘至恒重, 称 其干重, 然后计算地上部单株干物重及器官占单株 干重的比例。采用 WS Weather Station 仪(英国 Delta- $\mathrm{T}$ 公司)自动记录小麦生长季的逐日基本气象 资料。第 $i$ 天的累积有效积温 $G D D_{i}=\sum\left[\left(T M A X_{i}+\right.\right.$ $\left.T M I N_{\mathrm{i}}\right) / 2-T_{\mathrm{b}}$ ], 其中, $T M A X_{i}$ 和 $T M I N_{i}$ 为第 $i$ 天的最高 和最低气温, $T_{\mathrm{b}}$ 为基点温度 $\left(0^{\circ} \mathrm{C}\right)$ 。

\section{4 数据处理与分析}

1.4.1 数据归一化 将测得的不同生育时期的干 物质积累量 $D M A_{\mathrm{i}}$ 、器官干物质分配比例 $P_{\mathrm{i}}$ 和有效 积温 $G D D_{\mathrm{i}}$ 均进行归一化处理, 分别得到不同生育 时期的相对干物质积累量 $D M A_{R}$ 、相对器官分配比 例 $P_{\mathrm{R}}$ 和相对有效积温 $G D D_{\mathrm{R}}, D M A_{\mathrm{R}}=D M A_{\mathrm{i}} / D M A_{\mathrm{m}}$, $G D D_{\mathrm{R}}=G D D_{\mathrm{i}} / G D D_{\mathrm{m}}, P_{\mathrm{R}}=P_{\mathrm{i}} / P_{\mathrm{m}}$ 。其中 $D M A_{\mathrm{m}}$ 为最大 干物质积累量、 $P_{\mathrm{m}}$ 为器官分配比例最大值、 $G D D_{\mathrm{m}}$ 为收获时累积有效积温。

\subsection{2 数学方程拟合 基于归一化数据, 对各处} 理 3 次重复数据取均值, 然后使用 SAS 中的 Proc nlin 模块进行模型拟合及参数估计 ${ }^{[25]}$ 。利用残差平 方和简化测验(sum of square reduction test, SSRT) ${ }^{[26]}$ 比较处理间差异。因两年两地点试验结果趋势基本 一致, 以较为详细的 2007-2008 年获取的数据进行 分析。
以 $G D D_{\mathrm{R}}$ 为自变量 $(x), D M A_{\mathrm{R}}$ 为因变量 $(y)$, 采用 Richards 方程 $y=A /(1+b \times \exp (-k \times x))^{1 / N}$ 进行干物质积 累动态的拟合。其中, $A$ 为终极生长量, $b$ 为初值参数, $k$ 为生长速率参数, $N$ 为形状参数。次级特征参数如 下。起始生长势 $R_{0}=k / N$; 平均生长速率 $R_{\mathrm{a}}=$ $A \times k /(2 N+4)$; 生长活跃期 $D=(2 N+4) / k$; 最大生长速 率 $R_{\text {max }}$ 到达时的相对 GDD，即 $G_{\text {Rmax }}=(\ln b-\ln N) / k$; 将 $G_{\mathrm{Rmax}}$ 代入 Richards 方程一阶导数中求得 $R_{\max }$; 于 $G_{\mathrm{R} \max }$ 时的生长量 $W_{\mathrm{R} \text { max }}=A /(N+1)^{1 / N}$; Richards 生长曲 线呈明显的三阶段增长趋势, 其中 0 至 $G D D_{1}$ 为渐增 期, 相应持续期 $D_{1}=G D D_{1} ; G D D_{1}$ 至 $G D D_{2}$ 为快增期, 相应持续期为 $D_{2}=G D D_{2}-G D D_{1} ; G D D_{2}$ 至 $G D D_{3}$ 为 缓增期, 相应持续期为 $D_{3}=G D D_{3}-G D D_{2}$ 。其中, $G D D_{1}=-\ln \left[N^{2}+3 N+N \times\left(N^{2}+6 N+5\right)^{0.5}\right] / k, G D D_{2}=-\ln$ $\left[N^{2}+3 N-N \times\left(N^{2}+6 N+5\right)^{0.5}\right] / k$; 生长终期 $G D D_{3}=[-\ln$ $\left.(100 A / 99)^{N}-1\right] / b / k$ 。具体推导过程见文献[20]。

根据器官分配动态曲线, 以 $G D D_{\mathrm{R}}$ 为自变量 $(x)$, $P_{\mathrm{R}}$ 为因变量 $(y)$, 采用 VP 方程 $y=\exp (a+b / x+c \times \ln x)$ 进行器官分配比例动态的拟合。对 VP 方程求一阶 和二阶导数, 得到 $y^{\prime}=\left(c / x-b / x^{2}\right) \times \exp (a+b / x+c \times \ln x)$, $y^{\prime \prime}=\left[2 b / x^{3}-c / x^{2}+\left(c / x-b / x^{2}\right)^{2}\right] \times \exp (a+b / x+c \times \ln x)$ 。叶和 穗分配比例变化曲线为单调递减和递增曲线, 其渐 近线峰值为 $y_{\max }=\exp [c \times \ln (b / c)+a+c]$ 。Darroch 和 Baker ${ }^{[27]}$ 认为, 理论上作物生长不能达到渐近线峰 值, 即达到渐近线峰值的 $95 \%$ 时生长完成, 所以叶、 穗最大分配比例 $P_{\max }$ 为 $0.95 \times y_{\max }$ 。令 $\mathrm{VP}$ 方程分别 为叶部和穗部 $P_{\text {max }}$, 求解得到叶和穗到达 $P_{\text {max }}$ 时的 相对 GDD, 即叶部 $G_{P \max }$ 和穗部 $G_{\mathrm{P} \text { max }}$; 茎鞘分配比 例变化曲线为单峰曲线, $G_{\mathrm{SP} \max }=b / c, P_{\mathrm{S} \max }=\exp [c \times \ln$ $(b / c)+a+c]$ 。令 $y^{\prime \prime}$ 为零, 求解得到达到最大增长速率 时的相对 GDD, 即 $G_{\text {Simax }}=\left[c-1+(1-c)^{0.5}\right] \times b /\left(c^{2}-c\right)$ 和 最大下降速率时的相对 GDD, 即 $G_{\text {Sdmax }}=[c-1-(1-$ $\left.c)^{0.5}\right] \times b /\left(c^{2}-c\right)$ 。把 $G_{S i m a x}$ 和 $G_{S d \max }$ 分别代入 $y^{\prime}$, 即求 得最大增长速率 $R_{\mathrm{Simax}}$ 和最大下降速率 $R_{\mathrm{Sdmax}}$ 。

\section{2 结果与分析}

\section{1 地上部干物质随生育进程的积累动态}

由图 1 可以看出, 生长前期植株干重增长缓慢, 拔节后增加迅速, 临近成熟期又增长缓慢, 总体呈 “慢一快一慢”的 S 形曲线。拔节前各处理干物质 积累量差异不明显, 拔节后处理间差异逐渐增大, 且单株干物重随施氮水平的提高而增加。

各拟合方程的决定系数均大于 $0.993, \mathrm{RMSE}$ 均 小于 0.1 (表 2), 表明 Richards 方程可较准确地描述 

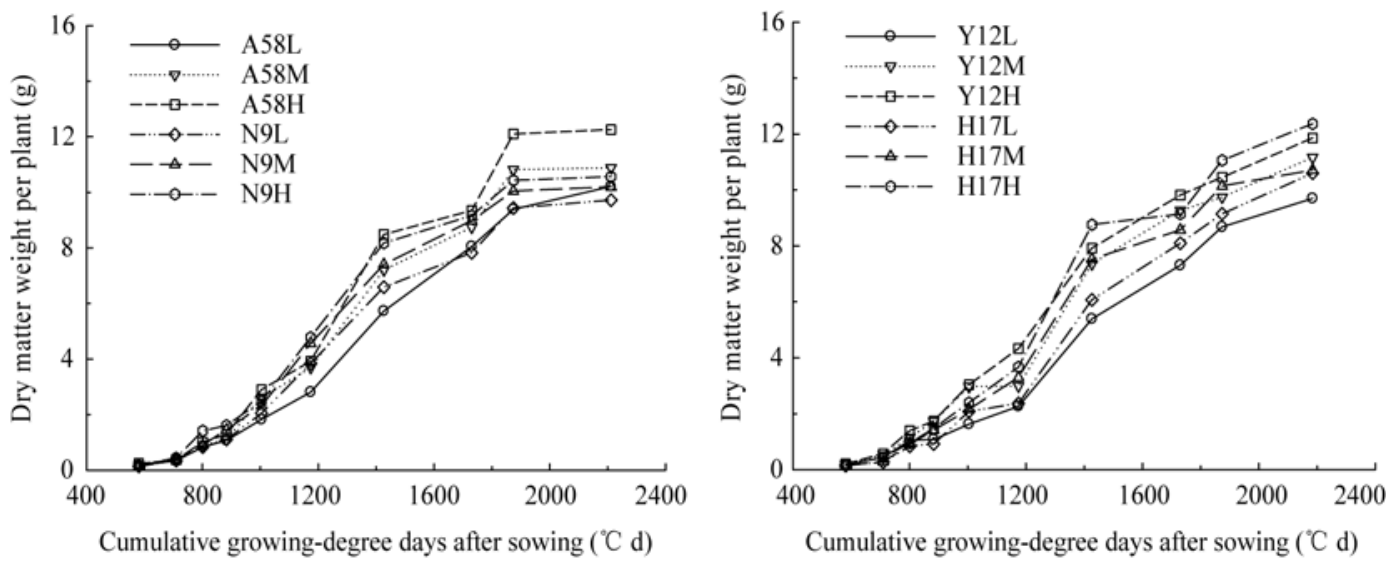

图 1 小麦单株干物质积累量随 GDD 的动态变化(2007-2008 生长季)

Fig. 1 Dynamic changes of dry matter accumulation per plant in wheat cultivars with growing-degree days (2007-2008 growing season)

A58: 矮抗 58: N9: 宁麦 9 号: H17: 淮麦 17; Y12: 扬麦 12。L: 施氮 $75 \mathrm{~kg} \mathrm{hm}^{-2}$ 处理; M: 施氮 $150 \mathrm{~kg} \mathrm{hm}^{-2}$ 处理; $\mathrm{H}$ : 施氮 $225 \mathrm{~kg} \mathrm{hm}^{-2}$ 处理。

A58: Aikang 58; N9: Ningmai 9; H17: Huaimai 17; Y12: Yangmai 12. L: nitrogen application rate of $75 \mathrm{~kg} \mathrm{hm}^{-2}$; M: nitrogen application rate of $150 \mathrm{~kg} \mathrm{hm}$; $\mathrm{H}$ : nitrogen application rate of $225 \mathrm{~kg} \mathrm{hm}^{-2}$.

表 2 小麦单株干物质积累动态拟合方程参数及特征参数(2007-2008 生长季)

Table 2 Parameters of Richards equation on dry matter accumulation in wheat cultivars (2007-2008 growing season)

\begin{tabular}{|c|c|c|c|c|c|c|c|c|c|c|c|c|c|c|c|}
\hline \multirow{2}{*}{$\begin{array}{c}\text { 处理 } \\
\text { Treatment }\end{array}$} & \multicolumn{4}{|c|}{ 方程参数 Equation parameter } & \multirow{2}{*}{$R^{2}$} & \multirow{2}{*}{ RMSE } & \multicolumn{9}{|c|}{ 特征参数 Characteristic parameter } \\
\hline & $A$ & $b$ & $k$ & $N$ & & & $R_{0}$ & $R_{\mathrm{a}}$ & $D$ & $\overline{G_{\mathrm{R} \max }}$ & $W_{\mathrm{R} \max }$ & $R_{\max }$ & $D_{1}$ & $D_{2}$ & $D_{3}$ \\
\hline \multicolumn{16}{|c|}{ 宁麦 9 号 Ningmai 9} \\
\hline N75 & 1.062 & 2.553 & 6.288 & 0.085 & $0.998^{* *}$ & 0.032 & 74.35 & 1.60 & 0.66 & 0.54 & 0.41 & 2.36 & 0.38 & 0.32 & 0.26 \\
\hline N150 & 1.029 & 21.687 & 8.051 & 0.299 & $0.999^{* *}$ & 0.051 & 26.97 & 1.80 & 0.57 & 0.53 & 0.43 & 2.66 & 0.40 & 0.27 & 0.27 \\
\hline N225 & 1.004 & 110.000 & 9.646 & 0.613 & $0.998^{* *}$ & 0.024 & 15.73 & 1.85 & 0.54 & 0.54 & 0.46 & 2.75 & 0.41 & 0.25 & 0.32 \\
\hline \multicolumn{16}{|c|}{ 矮抗 58 Aikang 58} \\
\hline N75 & 1.061 & 72.617 & 7.644 & 0.634 & $0.997^{* *}$ & 0.033 & 12.06 & 1.54 & 0.69 & 0.62 & 0.49 & 2.29 & 0.46 & 0.32 & 0.19 \\
\hline N150 & 1.062 & 15.823 & 7.045 & 0.303 & $0.993^{* *}$ & 0.019 & 23.24 & 1.62 & 0.65 & 0.56 & 0.44 & 2.40 & 0.41 & 0.31 & 0.22 \\
\hline N225 & 1.058 & 10.117 & 6.761 & 0.235 & $0.994^{* *}$ & 0.034 & 28.77 & 1.60 & 0.66 & 0.56 & 0.43 & 2.36 & 0.40 & 0.31 & 0.24 \\
\hline \multicolumn{16}{|c|}{ 淮麦 17 Huaimai 17} \\
\hline N75 & 1.094 & 14.229 & 6.094 & 0.322 & $0.997^{* *}$ & 0.037 & 18.94 & 1.44 & 0.76 & 0.62 & 0.46 & 2.12 & 0.44 & 0.36 & 0.20 \\
\hline $\mathrm{N} 150$ & 1.021 & 51.269 & 8.119 & 0.453 & $0.996^{* *}$ & 0.097 & 17.93 & 1.69 & 0.60 & 0.58 & 0.45 & 2.50 & 0.44 & 0.28 & 0.29 \\
\hline N225 & 1.039 & 3.964 & 6.204 & 0.124 & $0.993^{* *}$ & 0.060 & 50.17 & 1.52 & 0.68 & 0.56 & 0.40 & 2.24 & 0.40 & 0.33 & 0.32 \\
\hline \multicolumn{16}{|c|}{ 扬麦 12 Yangmai 12} \\
\hline N75 & 1.074 & 91.579 & 7.409 & 0.739 & $0.998^{* *}$ & 0.049 & 10.02 & 1.45 & 0.74 & 0.65 & 0.51 & 2.16 & 0.48 & 0.33 & 0.17 \\
\hline N150 & 1.034 & 45.266 & 7.338 & 0.593 & $0.995^{* *}$ & 0.042 & 12.38 & 1.46 & 0.71 & 0.59 & 0.47 & 2.17 & 0.43 & 0.32 & 0.26 \\
\hline N225 & 1.069 & 1.698 & 5.549 & 0.084 & $0.999^{* *}$ & 0.058 & 65.87 & 1.42 & 0.75 & 0.54 & 0.41 & 2.09 & 0.36 & 0.36 & 0.28 \\
\hline
\end{tabular}

RMSE：根均方差; $R_{0}$ ：起始生长势; $R_{\mathrm{a}}$ ：平均生长速率; $D$ : 生长活跃期; $R_{\text {max }}$ ：最大生长速率; $G_{\mathrm{R} \text { max }}$ ：达到 $R_{\text {max }}$ 时的相对 $\mathrm{GDD}$; $W_{\mathrm{Rmax}}$ ：达到 $R_{\text {max }}$ 时的相对生长量; $D_{1}$ ：渐增持续期; $D_{2}$ ：快增持续期; $D_{3}$ ：缓增持续期。

RMSE: root mean square error; $R_{0}$ : initial growth potential; $R_{\mathrm{a}}$ : average growth rate; $D$ : active growth period; $R_{\max }$ : maximum growth rate; $G_{R \max }$ : relative growing-degree days at $R_{\max } ; W_{\mathrm{Rmax}}$ : relative dry matter accumulation at $R_{\max } ; D_{1}$ : relative duration of early stage; $D_{2}$ : relative duration of middle stage; $D_{3}$ : relative duration of last stage.

相对干物质积累量随相对 GDD 的变化动态。SSRT 检验表明, 方程参数在不同施氮水平和不同株型之 间具有显著差异(施氮量 $F$ 值为 $13.250, P<0.01$; 株 型 $F$ 值为 $8.536, P<0.01)$ 。供试株型的 $N$ 值均小于 1 , 说明出苗到拔节干物质增重缓慢。随施氮量的增 加, 矮抗 58 和扬麦 12 的 $R_{0}$ 逐渐增加, 宁麦 9 号的
$R_{0}$ 逐渐下降，而淮麦 17 的 $R_{0}$ 以 $\mathrm{N} 150$ 最低，说明适 量施氮有利于提高部分株型的 $R_{0}$, 从而缩短返青时 间。随施氮量的增加，宁麦 9 号、矮抗 58 和淮麦 17 的平均生长速率 $R_{\mathrm{a}}$ 呈上升趋势, 而扬麦 12 的 $R_{\mathrm{a}}$ 呈 先增加后下降的趋势, 说明施氮可提高 $R_{\mathrm{a}}$, 但施氮 量超过 N150 会造成部分株型的 $R_{\mathrm{a}}$ 下降。矮抗 58、 
淮麦 17 和扬麦 12 均以 $\mathrm{N} 150$ 和 $\mathrm{N} 225$ 达到 $R_{\text {max }}$ 所需 GDD 较少, 而宁麦 9 号以 N150 所需 GDD 最少, 说 明施氮促使 $R_{\max }$ 提早到达。矮抗 58 、淮麦 17 和扬 麦 12 的 $W_{\mathrm{Rmax}}$ 随施氮量的增加而逐渐下降, 而宁麦 9 号正好相反。供试株型的 $R_{\max }$ 均以 N150 和 N225 处理大于 $\mathrm{N} 75$, 说明施氮提高了 $R_{\max }$ 。从 $D_{1} 、 D_{2}$ 和 $D_{3}$ 来看, 供试株型均以 $D_{1}$ 最长。施氮延长了各株型 的缓增期 $D_{3}$, 但缩短了矮抗 58 、淮麦 17 和扬麦 12 的渐增期 $D_{1}$, 却延长宁麦 9 号的渐增期 $D_{1}$ 。宁麦 9 号和矮抗 58 的快增期 $D_{2}$ 均随施氮量增加呈下降趋 势, 而淮麦 17 和扬麦 12 均以 N150 最低。这说明施 氮可缩短矮抗 58、淮麦 17 和扬麦 12 的渐增期, 延 长各株型的缓增期, 但对快增持续期因品种各异。

\section{2 地上部干物质在不同器官中的分配动态}

从出苗到成熟，地上部干物质在叶片中的分配 比例随 GDD 的增加逐渐下降(图 2)。从出苗到拔节, 地上部干物质在茎鞘中的分配比例逐渐增加, 拔节 后分配比例的增加速率加快, 于抽穗时到达最大值, 随后又逐渐下降直至成熟。从抽穗到成熟, 地上部 干物质在穗部的分配比例呈不断上升趋势。根据上 述的变化趋势, 对地上部干物质在叶片、茎鞘和穗 部的相对分配比例用 VP 方程进行拟合。

拟合方程的拟合度均达到极显著水平，且 RMSE 小于 0.1 , 表明器官干物重分配比例随 GDD 的变化动态符合 VP 模型(表 3)。SSRT 检验表明, 叶、 穗分配比例的方程参数在不同株型、施氮水平之间 存在显著差异(数据未列出)。施氮延迟了淮麦 17 和 扬麦 12 到达叶部 $P_{\text {max }}$ 和 $R_{\mathrm{LEmax}}$ 的时间, 而缩短了矮 抗 58 和宁麦 9 号到达叶部 $P_{\text {max }}$ 和 $R_{\mathrm{LEmax}}$ 的时间。施
氮提高了宁麦 9 号和矮抗 58 的叶部 $P_{\text {max }}$, 而降低了 淮麦 17 和扬麦 12 的叶部 $P_{\max }$, 说明出苗后氮素对 宁麦 9 号和矮抗 58 叶片生长的促进作用大于其他品 种。施氮降低了矮抗 58 、淮麦 17 和扬麦 12 的叶部 $R_{\mathrm{LEmax}}$, 增加了宁麦 9 号的叶部 $R_{\mathrm{LEmax}}$ 。宁麦 9 号和 矮抗 58 的穗部 $G_{\mathrm{Pmax}} 、 P_{\max } 、 G_{\max }$ 和 $R_{\mathrm{LEmax}}$ 对氮素的 响应表现出相同趋势。施氮使宁麦 9 号和矮抗 58 延 迟了穗部 $P_{\text {max }}$ 到达时间, 且提高了穗部 $P_{\text {max }}$; 提前 了穗部 $R_{\mathrm{LEmax}}$ 到达时间, 但降低了穗部 $R_{\mathrm{LEmax}}$ 。施氮 对淮麦 17 和扬麦 12 与对宁麦 9 号和矮抗 58 的影响 有明显差异, 且淮麦 17 与扬麦 12 的变化趋势正好 相反。适量施氮使淮麦 17 的穗部 $P_{\max }$ 延迟, 而使扬 麦 12 的穗部 $P_{\max }$ 提前, 但过量施氮均产生负效应。 适量施氮提高了淮麦 17 的穗部 $P_{\max }$, 过量则降低穗 部 $P_{\max }$, 而施氮降低了扬麦 12 的穗部 $P_{\max }$ 。施氮使 淮麦 17 的穗部 $R_{\mathrm{LEmax}}$ 延迟, 但提高了穗部 $R_{\mathrm{LEmax}}$, 而 施氮提前扬麦 12 的穗部 $R_{\mathrm{LEmax}}$, 但扬麦 12 的穗部 $R_{\mathrm{LEmax}}$ 随施氮量呈先增加后下降的趋势。

拟合方程的拟合度均达到极显著水平，且 RMSE 较小。SSRT 检验表明, 茎鞘拟合方程参数在 不同株型小麦品种和施氮水平之间存在显著差异 (施氮量 $F$ 值为 $7.798, P<0.001$; 株型 $F$ 值为 4.599 , $P<0.001)$ 。从 $G_{\mathrm{SP} \max } 、 G_{\mathrm{Simax}}$ 和 $G_{\mathrm{Sdmax}}$ 来看, 施氮使 淮麦 17 和扬麦 12 的 $R_{\text {Simax }}$ 提早到达，而延迟了 $P_{\text {Smax }}$ 和 $R_{\mathrm{Sdmax}}$ 到达时间。施氮对宁麦 9 号和矮抗 58 的 $G_{\mathrm{SPmax}}$ 均呈先延迟后缩短的趋势, 而对 $G_{\mathrm{Simax}}$ 均呈持 续增加的趋势, 但对于 $G_{\mathrm{Sdmax}}$ 两株型表现正好相反, 宁麦 9 号先延迟后缩短, 而矮抗 58 持续下降(表 4)。 这说明施氮对紧凑型品种宁麦 9 号和矮抗 58 的 $P_{\text {Smax }}$
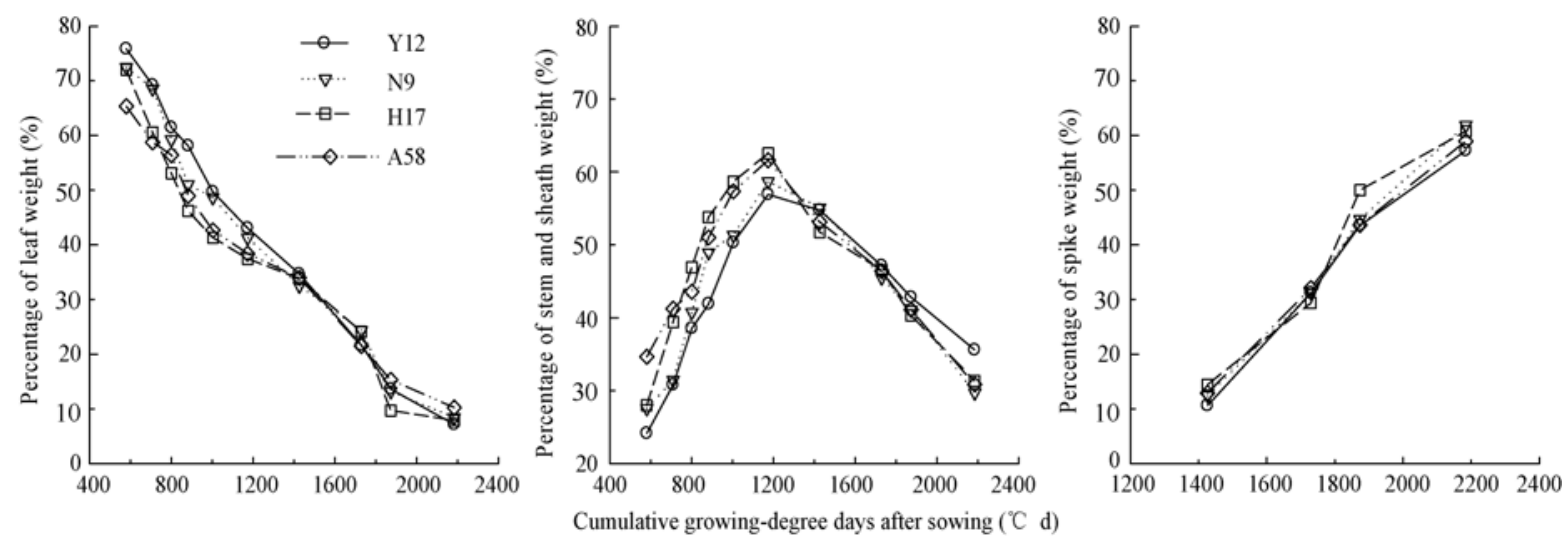

图 2 不同器官占小麦单株干物重比例的动态变化(2007-2008 生长季)

Fig. 2 Dynamic changes of ratio of organs dry weight to plant dry weight in wheat cultivars (2007-2008 growing season) 数据点为 3 个施氮处理的均值。

Each point is the average value of three nitrogen treatments. 
表 3 小麦叶穗器官干物质分配比例的拟合方程参数及特征参数(2007-2008 生长季)

Table 3 Parameters of VP equation for dry matter partitioning percentage to leaf and spike in wheat cultivars (2007-2008 growing season)

\begin{tabular}{|c|c|c|c|c|c|c|c|c|c|c|}
\hline \multirow{2}{*}{$\begin{array}{c}\text { 品种 } \\
\text { Cultivar }\end{array}$} & \multirow{2}{*}{$\begin{array}{c}\text { 处理 } \\
\text { Treatment }\end{array}$} & \multicolumn{3}{|c|}{ 方程参数 Equation parameter } & \multirow{2}{*}{$R^{2}$} & \multirow{2}{*}{ RMSE } & \multicolumn{4}{|c|}{ 特征参数 Characteristic parameter } \\
\hline & & $a$ & $b$ & $c$ & & & $G_{\mathrm{Pmax}}$ & $P_{\max }$ & $G_{\max }$ & $R_{\mathrm{LEmax}}{ }^{\dagger}$ \\
\hline \multicolumn{11}{|l|}{ 叶 Leaf } \\
\hline 宁麦 9 号 & N75 & -0.719 & -0.767 & -2.703 & $0.995^{* *}$ & 0.056 & 0.347 & 0.934 & 0.431 & 1.715 \\
\hline \multirow[t]{2}{*}{ Ningmai 9} & N150 & -1.092 & -0.744 & -2.936 & $0.995^{* *}$ & 0.061 & 0.308 & 0.950 & 0.381 & 2.082 \\
\hline & $\mathrm{N} 225$ & -1.207 & -0.805 & -3.208 & $0.992^{* *}$ & 0.059 & 0.302 & 0.970 & 0.373 & 2.303 \\
\hline 矮抗 58 & N75 & -0.847 & -0.929 & -3.252 & $0.994^{* *}$ & 0.057 & 0.343 & 0.928 & 0.424 & 1.955 \\
\hline \multirow[t]{2}{*}{ Aikang 58} & N150 & -1.100 & -0.478 & -2.185 & $0.990^{* *}$ & 0.052 & 0.274 & 0.984 & 0.341 & 1.974 \\
\hline & $\mathrm{N} 225$ & -0.969 & -0.437 & -1.960 & $0.996^{* *}$ & 0.067 & 0.283 & 0.960 & 0.353 & 1.728 \\
\hline 淮麦 17 & N75 & -1.276 & -0.604 & -2.684 & $0.992^{* *}$ & 0.066 & 0.275 & 0.993 & 0.342 & 2.287 \\
\hline \multirow{2}{*}{ Huaimai 17} & N150 & -1.236 & -0.478 & -2.267 & $0.990^{* *}$ & 0.073 & 0.263 & 0.977 & 0.327 & 2.098 \\
\hline & $\mathrm{N} 225$ & -1.129 & -0.550 & -2.403 & $0.989^{* *}$ & 0.076 & 0.284 & 0.960 & 0.353 & 1.986 \\
\hline 扬麦 12 & N75 & -1.187 & -0.523 & -2.385 & $0.994^{* *}$ & 0.063 & 0.272 & 0.995 & 0.339 & 2.136 \\
\hline \multirow[t]{2}{*}{ Yangmai 12} & $\mathrm{~N} 150$ & -0.938 & -1.017 & -3.574 & $0.994^{* *}$ & 0.078 & 0.339 & 0.932 & 0.417 & 2.121 \\
\hline & $\mathrm{N} 225$ & -0.946 & -0.840 & -3.069 & $0.994^{* *}$ & 0.052 & 0.330 & 0.915 & 0.409 & 1.925 \\
\hline \multicolumn{11}{|l|}{ 穗部 Spike } \\
\hline 宁麦 9 号 & N75 & 19.534 & -19.531 & -19.886 & $0.999^{* *}$ & 0.086 & 1.056 & 0.956 & 0.767 & 3.778 \\
\hline \multirow[t]{2}{*}{ Ningmai 9} & N150 & 17.502 & -17.490 & -17.567 & $0.992^{* *}$ & 0.038 & 1.075 & 0.962 & 0.765 & 3.596 \\
\hline & $\mathrm{N} 225$ & 13.098 & -13.080 & -12.426 & $0.986^{* *}$ & 0.093 & 1.154 & 0.984 & 0.765 & 3.121 \\
\hline 矮抗 58 & N75 & 14.490 & -14.496 & -14.132 & $0.998^{* *}$ & 0.013 & 1.119 & 0.948 & 0.762 & 3.211 \\
\hline \multirow[t]{2}{*}{ Aikang 58} & $\mathrm{~N} 150$ & 12.372 & -12.367 & -12.134 & $0.999^{* *}$ & 0.048 & 1.119 & 0.957 & 0.738 & 3.115 \\
\hline & $\mathrm{N} 225$ & 11.181 & -11.170 & -10.740 & $0.995^{* *}$ & 0.064 & 1.148 & 0.969 & 0.737 & 2.983 \\
\hline 淮麦 17 & N75 & 10.519 & -10.505 & -9.748 & $0.990^{* *}$ & 0.053 & 1.196 & 0.991 & 0.749 & 2.867 \\
\hline \multirow{2}{*}{ Huaimai 17} & $\mathrm{~N} 150$ & 10.947 & -10.938 & -9.991 & $0.995^{* *}$ & 0.036 & 1.216 & 0.998 & 0.765 & 2.867 \\
\hline & $\mathrm{N} 225$ & 22.898 & -22.878 & -23.529 & $0.983^{* *}$ & 0.725 & 1.040 & 0.978 & 0.776 & 4.137 \\
\hline & N75 & 13.880 & -13.879 & -12.879 & $0.998^{* *}$ & 0.040 & 1.181 & 0.986 & 0.788 & 3.089 \\
\hline \multirow{2}{*}{ Yangmai 12} & N150 & 15.550 & -15.541 & -15.312 & $0.996^{* *}$ & 0.018 & 1.102 & 0.961 & 0.764 & 3.371 \\
\hline & $\mathrm{N} 225$ & 10.396 & -10.399 & -9.432 & $0.998^{* *}$ & 0.039 & 1.226 & 0.992 & 0.761 & 2.783 \\
\hline
\end{tabular}

RMSE：根均方差; $a 、 b 、 c$ : 方程参数; $P_{\text {max }}$ : 最大干物质分配比例; $G_{\mathrm{P} \text { max }}$ 到达 $P_{\text {max }}$ 时的相对 GDD; $R_{\mathrm{LEmax}}$ : 分配比例最大变化速 率; $G_{\max }$ ：到达 $R_{\mathrm{LEmax}}$ 时的相对 $\mathrm{GDD}$ 。 叶部 $R_{\mathrm{LEmax}}$ 为最大下降速率，穗部 $R_{\mathrm{LEmax}}$ 为最大增长速率。

RMSE: root mean square error; $a, b$, and $c$ : equation parameters; $P_{\max }$ : maximum dry matter partitioning percentage; $G_{\mathrm{Pmax}}$ : relative growing-degree days at $P_{\max } ; R_{\mathrm{LEmax}}$ : maximum changing rate of dry matter partitioning percentage; $G_{\max }$ : relative growing-degree days at $R_{\mathrm{LEmax} .} \cdot{ }^{\dagger} R_{\mathrm{LEmax}}$ for leaf and spike mean maximum decreasing and increasing rate, respectively.

表 4 小麦茎鞘干物重分配比例的模拟方程参数及特征参数(2007-2008 生长季)

Table 4 Parameters of VP equation for dry matter partitioning percentage to stem and sheath in wheat cultivars (2007-2008 growing season)

\begin{tabular}{|c|c|c|c|c|c|c|c|c|c|c|c|c|}
\hline \multirow{2}{*}{$\begin{array}{c}\text { 品种 } \\
\text { Cultivar }\end{array}$} & \multirow{2}{*}{$\begin{array}{c}\text { 处理 } \\
\text { Treatment }\end{array}$} & \multirow{2}{*}{$\frac{\text { 方程参数 }}{a_{\mathrm{S}}}$} & \multicolumn{2}{|c|}{ Equation parameter } & \multirow{2}{*}{$R^{2}$} & \multirow{2}{*}{ RMSE } & \multicolumn{6}{|c|}{ 特征参数 Characteristic parameter } \\
\hline & & & $b_{\mathrm{S}}$ & $c_{\mathrm{S}}$ & & & $G_{\mathrm{SPmax}}$ & $P_{\text {Smax }}$ & $G_{\text {Simax }}$ & $G_{\text {Sdmax }}$ & $R_{\text {Simax }}$ & $R_{\text {sdmax }}$ \\
\hline 宁麦 9 号 & N75 & 0.803 & -1.296 & -2.471 & $0.991^{* *}$ & 0.061 & 0.524 & 0.930 & 0.243 & 0.806 & 4.192 & 0.634 \\
\hline \multirow[t]{2}{*}{ Ningmai 9} & N150 & 1.354 & -1.900 & -3.428 & $0.992^{* *}$ & 0.062 & 0.554 & 0.950 & 0.291 & 0.817 & 4.150 & 0.751 \\
\hline & $\mathrm{N} 225$ & 1.108 & -1.802 & -3.462 & $0.989^{* *}$ & 0.063 & 0.520 & 0.911 & 0.274 & 0.767 & 4.243 & 0.676 \\
\hline 矮抗 58 & N75 & 0.611 & -1.085 & -2.146 & $0.993^{* *}$ & 0.089 & 0.506 & 0.931 & 0.221 & 0.791 & 4.335 & 0.589 \\
\hline \multirow[t]{2}{*}{ Aikang 58} & N150 & 0.917 & -1.539 & -3.009 & $0.987^{* *}$ & 0.077 & 0.511 & 0.928 & 0.256 & 0.767 & 4.339 & 0.661 \\
\hline & $\mathrm{N} 225$ & 0.616 & -1.304 & -2.743 & $0.993^{* *}$ & 0.091 & 0.475 & 0.917 & 0.230 & 0.721 & 4.579 & 0.603 \\
\hline 淮麦 17 & N75 & 1.019 & -1.726 & -3.442 & $0.996^{* *}$ & 0.056 & 0.502 & 0.953 & 0.264 & 0.739 & 4.602 & 0.677 \\
\hline \multirow[t]{2}{*}{ Huaimai 17} & N150 & 0.973 & -1.588 & -3.147 & $0.997^{* *}$ & 0.051 & 0.505 & 0.979 & 0.257 & 0.752 & 4.655 & 0.694 \\
\hline & $\mathrm{N} 225$ & 0.979 & -1.616 & -3.119 & $0.985^{* *}$ & 0.073 & 0.518 & 0.915 & 0.263 & 0.773 & 4.236 & 0.664 \\
\hline 扬麦 12 & N75 & 1.404 & -2.031 & -3.776 & $0.990^{* *}$ & 0.080 & 0.538 & 0.970 & 0.292 & 0.784 & 4.411 & 0.756 \\
\hline \multirow[t]{2}{*}{ Yangmai 12} & $\mathrm{~N} 150$ & 1.053 & -1.392 & -2.347 & $0.995^{* *}$ & 0.102 & 0.593 & 0.934 & 0.269 & 0.917 & 3.715 & 0.641 \\
\hline & $\mathrm{N} 225$ & 0.908 & -1.303 & -2.241 & $0.995^{* *}$ & 0.075 & 0.582 & 0.888 & 0.259 & 0.905 & 3.597 & 0.592 \\
\hline
\end{tabular}

RMSE: 根均方差; $a_{\mathrm{S}} 、 b_{\mathrm{S}} 、 c_{\mathrm{S}}$ : 茎鞘方程参数; $P_{\mathrm{S} \max }$ ：茎鞘最大分配比例; $G_{\mathrm{SP} \max }$ ：到达 $P_{\mathrm{S} \max }$ 时的相对 $\mathrm{GDD} ; R_{\mathrm{Simax}}$ ：茎鞘分配比例 最大增长速率; $G_{\mathrm{S} \text { Simax }}$ ：到达 $R_{\mathrm{S} \text { Simax }}$ 时的相对 GDD; $R_{\mathrm{Sdmax}}$ ：茎鞘分配比例最大下降速率; $G_{\mathrm{Sdmax}}$ ：到达 $R_{\mathrm{Sdmax}}$ 时的相对 GDD。

RMSE: root mean square error; $a_{\mathrm{S}}, b_{\mathrm{S}}, c_{\mathrm{S}}$ : equation parameters of stem and sheath; $P_{\mathrm{Smax}}$ : maximum dry matter partitioning percentage to stem and sheath; $G_{\mathrm{SPmax}}$ : relative growing-degree days at $P_{\mathrm{S} \max } ; R_{\mathrm{Simax}}$ : maximum increasing rate of dry matter partitioning percentage to stem and sheath; $G_{\text {Simax }}$ : relative growing-degree days at $R_{\text {Simax }} ; R_{\text {Sdmax }}$ : maximum decreasing rate of dry matter partitioning percentage to stem and sheath; $G_{\text {Sdmax }}$ : relative growing-degree days at $R_{\text {Sdmax }}$. 
和 $R_{\mathrm{Simax}}$ 影响一致, 而对 $R_{\mathrm{Sdmax}}$ 的影响因品种而异。 宁麦 9 号和淮麦 17 的 $P_{\mathrm{S} \max }$ 为 $\mathrm{N} 150>\mathrm{N} 75>\mathrm{N} 225$, 而 矮抗 58 和扬麦 12 的 $P_{\mathrm{Smax}}$ 为 $\mathrm{N} 225>\mathrm{N} 150>\mathrm{N} 75$, 说 明施氮降低了矮抗 58 和扬麦 12 的茎鞘分配最大比 例, 即增加了叶和穗的分配比例。适量施氮提高了 宁麦 9 号和淮麦 17 的 $P_{\text {Smax }}$, 即相应减少了叶和穗的 分配比例, 但过量施氮可降低其 $P_{S \max }$ 。施氮降低了 扬麦 12 的茎鞘分配比例的增长和下降速率, 而适量 施氮可提高淮麦 17 茎鞘分配比例的增长和下降速率, 但过量施氮则与中间型品种表现一致。随施氮量的 增加, 宁麦 9 号和矮抗 58 的 $R_{\mathrm{Sdmax}}$ 均呈先增加而后 下降的趋势, 而对于 $R_{\text {Simax }}$ 两株型表现不一致, 宁 麦 9 号呈先下降后增加趋势, 而矮抗 58 的 $R_{\text {Simax }}$ 持 续增加。这说明适量施氮加速了宁麦 9 号茎鞘分 配比例的下降, 而促进了矮抗 58 的茎鞘分配比例的 增长。

\section{3 讨论}

生长函数可解释作物生长曲线形状, 且推导出 的特征参数具有生物意义, 可用于比较处理效应, 因此农学家对干物质积累动态的方程拟合进行了许 多研究 ${ }^{[7-9]}$ 。本研究表明, Richards 和 VP 方程分别能 较准确地拟合干物质积累和器官分配比例动态。根 据推导出的特征参数, 从出苗到成熟期, 干物质积 累动态可分为出苗到拔节(渐增期)、拔节到抽穗开花 (快增期)和抽穗开花到成熟(缓增期) 3 个阶段, 其中 $R_{\max }$ 出现于孕穗期至开花期, 与周竹青 ${ }^{[17]}$ 、高翔等 ${ }^{[28]}$ 的结果基本一致。在 3 个阶段中, 渐增期 $D_{1}$ 的干物 质增长缓慢且持续时间最长, 通过适量施氮可起到 提高起始生长势, 促进渐增期干物质生产的作用, 但对宁麦 9 号却有负效应。适量施氮能提高 $R_{\mathrm{a}}$ 和 $R_{\max }$, 但超过一定施氮量会对其产生抑制作用。随生育进 程的推进, 小麦干物质分配中心不断发生变化。本 研究表明, 供试品种的叶片最大分配比例 $P_{\text {max }}$ 出现 于出苗到返青期, 而茎鞘的最大分配比例 $P_{\mathrm{S} \max }$ 出现 于孕穗期, 穗部的最大分配比例 $P_{\text {max }}$ 出现于成熟期, 与王高武等 ${ }^{[29]}$ 和杨连新等 ${ }^{[30]}$ 的结果基本一致。施氮 有利于紧凑型品种宁麦 9 号和矮抗 58 叶干物质的积 累, 进而增加绿叶面积, 最终提高穗部的干物质积 累, 提高籽粒产量。而对于松散型淮麦 17 和中间型 扬麦 12 , 施氮有利于茎鞘分配比例的提高, 相对降 低了叶、穗的分配比例。氮素对不同株型小麦不同 器官最大变化速率的影响也存在明显差异。因此, 在培育高产群体时, 应该充分考虑到株型因素, 针
对不同株型采用不同培育途径，与封超年等 ${ }^{[6]}$ 提出 的品种一栽培措施一群体一株型一产量主线基本相 同。

研究表明，小麦的干物质积累动态呈“慢一快

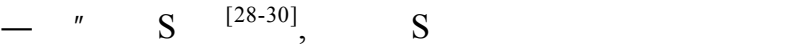
分歧。任正隆和李尧权 ${ }^{[12]}$ 、吴晓飞等 ${ }^{[31]}$ 认为小麦花 后到成熟期间的干物质变化趋势呈 $\mathrm{S}$ 形, 且使用 Logistic 方程进行拟合的效果较好 ${ }^{[17]}$ 。而高翔等 ${ }^{[28]}$ 则认为从出苗到成熟小麦干物质变化趋势呈 $\mathrm{S}$ 形, 且周竹青 ${ }^{[17]}$ 和张利等 ${ }^{[11]}$ 利用 Logistic 方程进行拟合 的效果较好。Sheehy 等 ${ }^{[13]}$ 认为水稻存在营养生长和 生殖生长阶段两个 $\mathrm{S}$ 形曲线, 并用 Logistic 曲线进行 了验证。本研究表明, 利用 Richards 模型对小麦整 个生育期的干物质积累动态拟合效果较好, 但利用 Richards 模型仅对开花到成熟期干物质积累动态进 行拟合, 其效果并不理想(数据未列出), 表明小麦花 后干物质积累动态可能不符合 $\mathrm{S}$ 形曲线, 这与高翔 等 ${ }^{[28]}$ 和周竹青 ${ }^{[17]}$ 的研究结果一致。Villegas 等 ${ }^{[15]}$ 曾 报道使用非对称 Logistic 单峰曲线拟合小麦干物质 积累动态，尽管该方程参数没有生物学意义，但由 方程参数导出的次级参数具有生物学意义。因此, 本研究尝试使用 VP 方程拟合小麦器官分配比例动 态，拟合度达到显著水平。该方程的次级特征参数 具有生物学意义, 适合单峰曲线的拟合, 但其拟合 方程的适用范围还有待今后进一步的探讨。

\section{4 结论}

Richards 和 VP方程可比较准确地拟合不同株型 品种和施氮条件下小麦全生育期干物质积累及其在 不同器官间的分配比例动态。不同株型小麦干物质 积累与分配对施氮量的响应差异主要表现在干物质 积累的起始生长势、渐增和快增持续期, 以及各器 官的最大分配比例、分配比例最大下降速率和最大 增长速率。

\section{References}

[1] Yao S-M(姚素梅), Kang Y-H(康跃虎), Liu H-J(刘海军). Studies on dry matter accumulation, partitioning and translocation in winter wheat under sprinkler and surface irrigation conditions. Agric Res Arid Areas (干旱地区农业研究), 2008, 26(6): 51-56 (in Chinese with English abstract)

[2] Latiri-Souki K, Nortcliff S, Lawlor D W. Nitrogen fertilizer can increase dry matter, grain production and radiation and water use efficiencies for durum wheat under semi-arid conditions. Eur $J$ Agron, 1998, 9: 21-34

[3] Olesen J E, Berntsen J, Hansen E M, Petersen B M, Petersen J. 
Crop nitrogen demand and canopy area expansion in winter wheat during vegetative growth. Eur J Agron, 2002, 16: 279-294

[4] Acreche M M, Briceno-Feix G, Martin S J A, Slafer G A. Radiation interception and use efficiency as affected by breeding in Mediterranean wheat. Field Crops Res, 2009, 110: 91-97

[5] Yunusa I A M, Siddique K H M, Belford R K, Karimi M M. Effect of canopy structure on efficiency of radiation interception and use in spring wheat cultivars during the pre-anthesis period in a Mediterranean-type environment. Field Crops Res, 1993, 35: 113-122

[6] Feng C-N(封超年), Guo W-S(郭文善), He J-H(何建华), Zhu $\mathrm{X}-\mathrm{K}$ (朱新开), Ma G-H(马光辉). The characteristics of plant type of high yield in wheat. J Yangzhou Univ (Nat Sci Edn) (扬州大学 学报·自然科学版), 1998, 1(4): 24-30 (in Chinese with English abstract)

[7] $\mathrm{Hu}$ Y-J(胡延吉), Lan J-H(兰进好), Zhao T-F(赵檀方). Dry matter accumulation and partitioning in three major wheat cultivars released in different period. J Shangdong Agric Univ (山 东农业大学学报), 1999, 30(4): 404-408 (in Chinese with English abstract)

[8] Arduini I, Masoni A, Ercoli L, Mariotti M. Grain yield, and dry matter and nitrogen accumulation and remobilization in durum wheat as affected by variety and seeding rate. Eur J Agron, 2006, 25: 309-318

[9] Dordas C. Dry matter, nitrogen and phosphorus accumulation, partitioning and remobilization as affected by $\mathrm{N}$ and $\mathrm{P}$ fertilization and source-sink relations. Eur J Agron, 2009, 30: 129-139

[10] Hunt R. Plant Growth Analysis. London: Edward Armold Publishers, 1982

[11] Zhang L(张利), Sui X-X(隋新霞), Wang Y(王羽), Fan Q-Q(㷊庆 琦), Li G-Y(李根英), Chu X-S(楚秀生), Huang C-Y(黄承彦). Dry matter accumulation and assignment rule of wheat cultivars with different maturity. Shangdong Agric Sci (山东农业科学), 2007, (6): 54-56 (in Chinese with English abstract)

[12] Ren Z-L(任正隆), Li Y-Q(李尧权). Varietal difference of filling rate and relative growth rate of wheat grain and dry matter accumulation after anthesis in wheat. Sci Agric Sin (中 国农业科学), 1981, 14(6): 12-20 (in Chinese with English abstract)

[13] Sheehy J E, Mitchell P L, Ferrer A B. Bi-phasic growth patterns in rice. Ann Bot, 2004, 94: 811-817

[14] Goudriaan J, Monteith J L. A mathematical function for crop growth based on light interception and leaf area expansion. Ann Bot, 1990, 66: 695-701

[15] Villegas D, Aparicio N, Blanco R, Royo C. Biomass accumulation and main stem elongation of durum wheat grown under Mediterranean conditions. Ann Bot, 2001, 88: 617-627

[16] Royo C, Blanco R. Growth analysis of five spring and five winter triticale genotypes. Agron J, 1999, 91: 305-311

[17] Zhou Z-Q(周竹青). Comparison of dry matter accumulaton and translocation of different wheat cultivars. Crops (作物杂志), 2002, (1): 16-19 (in Chinese)

[18] Qin S-H( 秦舒浩), Li L-L( 李玲玲). Accumulation and distribution of dry matter and grain filling of spring wheat postanthesis under supplementary irrigation catchment rainfall. $J$ Soil Water Conserv (水土保持学报), 2005, 19(4): 173-180 (in Chinese with English abstract)

[19] Xing L-F(邢黎峰), Sun M-G(孙明高), Wang Y-J(王元军). Richards growth model of living-organism. J Biomath (生物数学 学报), 1998, 13(3): 348-353 (in Chinese with English abstract)

[20] Zhu Q-S(朱庆森), Cao X-Z(曹显祖), Luo Y-Q(骆亦其). Growth analysis of the process of grain filling in rice. Acta Agron Sin (作 物学报), 1988, 14(3): 182-193 (in Chinese with Enlgish abstract)

[21] Yang J C, Zhang J H, Wang Z Q, Zhu Q S, Wang W. Hormonal changes in the grains of rice subjected to water stress during grain filling. Plant Physiol, 2001, 127: 315-323

[22] Yang Y-Q(杨义群), Wu L-H(吴良欢), Wu J-M(吴其苗). On the Richards curve. J Biomath (生物数学学报), 2000, 15(4): 385-387 (in English with Chinese abstract)

[23] Wang K-J(王空军), Zheng H-J(郑洪建), Liu K-C(刘开昌), Zhang J-W(张吉旺), Dong S-T(董树亭), Hu C-H(胡昌浩), Evolution of maize root distribution in space-time during maize varieties replacing in China. Acta Phytoecol Sin (植物生态学报), 2001, 25(4): 472-475 (in Chinese with English abstract)

[24] Faravani M, Bakar B H. Descriptive analysis on the morphology, growth and branching patterns of princess-flower (Tibouchina urvilleana Cogn.) in Peninsular Malaysia. J Food Agric Environ, 2007, 5: 234-238

[25] SAS Institute. SAS/STAT 9.1 User's Guide. Cary, NC, USA, 2004

[26] Schabenberger O, Pierce F J. Contemporary Statistical Models for the Plant and Soil Sciences. Boca Raton: Taylor \& Francis/ CRC Press, 2001

[27] Darroch B A, Baker R J. Grain filling in three spring wheat genotypes: statistical analysis. Crop Sci, 1990, 30: 525-529

[28] Guo X(高翔), Pang H-X(庞洪喜), Dong J(董剑). Studies on the accumulation and distribution of photosynthetic matter and character of grain-filling in high-yield wheat varieties. $J$ Northwest Sci-Tech Univ Agric For (Nat Sci Edn) (西北农林科 技大学学报·自然科学版), 2002, 30(4): 24-28 (in Chinese with English abstract)

[29] Wang G-W(王高武), Tang J-H(唐建华), Wu W-Z(吴维中). Characters of biomass production before and after heading and its relation to spike weight in wheat. Jiangsu J Agric Sci (江苏农业 科学), 1988, 4(2): 23-29 (in Chinese with English abstract)

[30] Yang L-X(杨连新), Wang Y-L(王余龙), Li S-F(李世峰), Huang J-H(黄建华), Dong G-C(董桂春), Zhu J-G(朱建国), Liu G(刘 钢), Han Y(韩勇). Effects of free-air $\mathrm{CO}_{2}$ enrichment (FACE) on dry matter production and allocation in wheat. Chin J Appl Ecol (应用生态学报), 2007, 18(2): 339-346 (in Chinese with English abstract)

[31] Wu X-F(吴晓飞), Shi S-B(石书兵), Ma L(马林), Meng F-D(孟凡 德), Zhu J(朱军), Mao J-X(毛吉贤), Guo F(郭飞), Wen X-X(闻小 霞). Effect of interplanting sweet clover under no-tillage and different stages on the dynamic change of dry matter accumulation of spring wheat. J Xinjiang Agric Univ (新疆农业大学学报), 2008, 31(1): 30-35 (in Chinese with English abstract) 